\title{
Linking structure and function in striatum using algebraic topology, digital microcircuit reconstruction and simulations of the healthy and diseased network
}

Ilaria Carannante ${ }^{1}$, Martina Scolamiero ${ }^{2}$, Alexander Kozlov ${ }^{1}$, Lihao Guo ${ }^{1}$, Johannes Hjorth ${ }^{1}$, Johanna Frost Nylén ${ }^{3}$, Joana Braga Pereira ${ }^{4}$, Arvind Kumar ${ }^{1}$, Wojciech Chachólski², Jeanette Hellgren Kotaleski ${ }^{1,3}$

\begin{abstract}
${ }^{1}$ KTH Royal Institute of Technology, Department of Computational Science and Technology, Solna, Sweden; ${ }^{2}$ KTH Royal Institute of Technology, Department of Mathematics, Stockholm, Sweden; ${ }^{3}$ Karolinska Institutet, Department of Neuroscience, Stockholm, Sweden;

${ }^{4}$ Karolinska Institute, Department of Neurobiology, Stockholm, Sweden
\end{abstract}

The relationship between the structure and network dynamics within the striatum is currently not well understood. We have applied algebraic topology to investigate the local structural connectivity in the striatum in healthy and at different stages of Parkinson's Disease (PD). We have then studied the consequences of cortico-striatal inputs loss in terms of signal transfer, especially correlation transfer.
A Basal ganglia

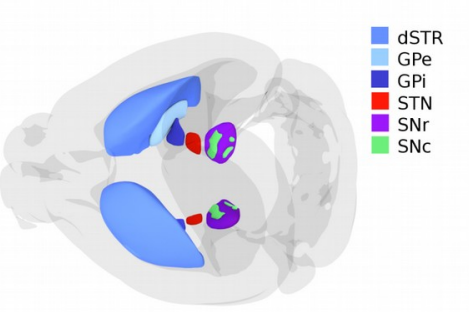

B

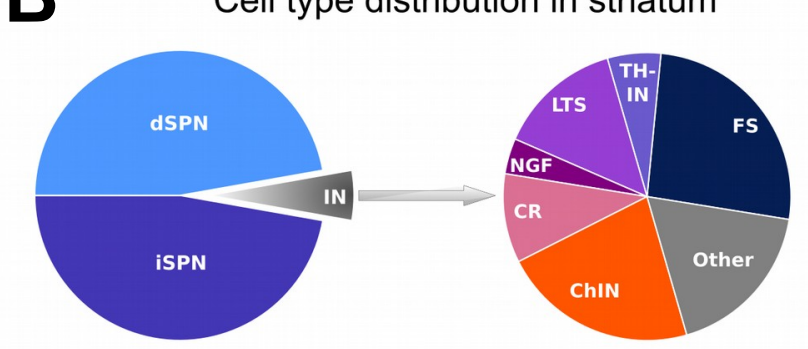

We created a full-scale digital reconstruction of the mouse striatal microcircuitry [1]. Using Snudda, neurons are randomly placed within the striatal volume reproducing realistic neuron densities and connectivities. An example of $300 \times 300 \times 300 \mu \mathrm{m}^{3}$ volume, consisting of 2174 neurons, is represented below.
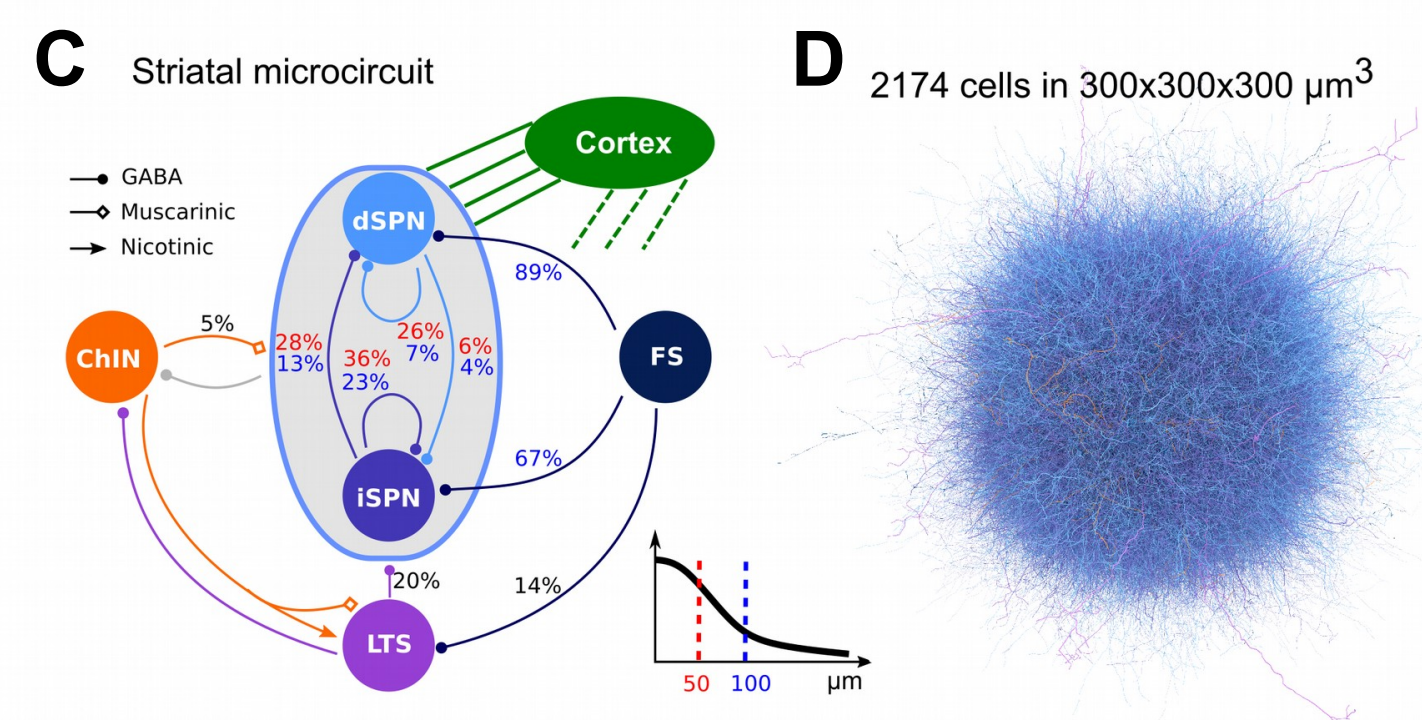

The PD stages are characterized by successively modified healthy morphologies of the striatal projection neurons (SPN) according to [2] and [3]. The removed red branches in the below panel represent the dendritic atrophy.

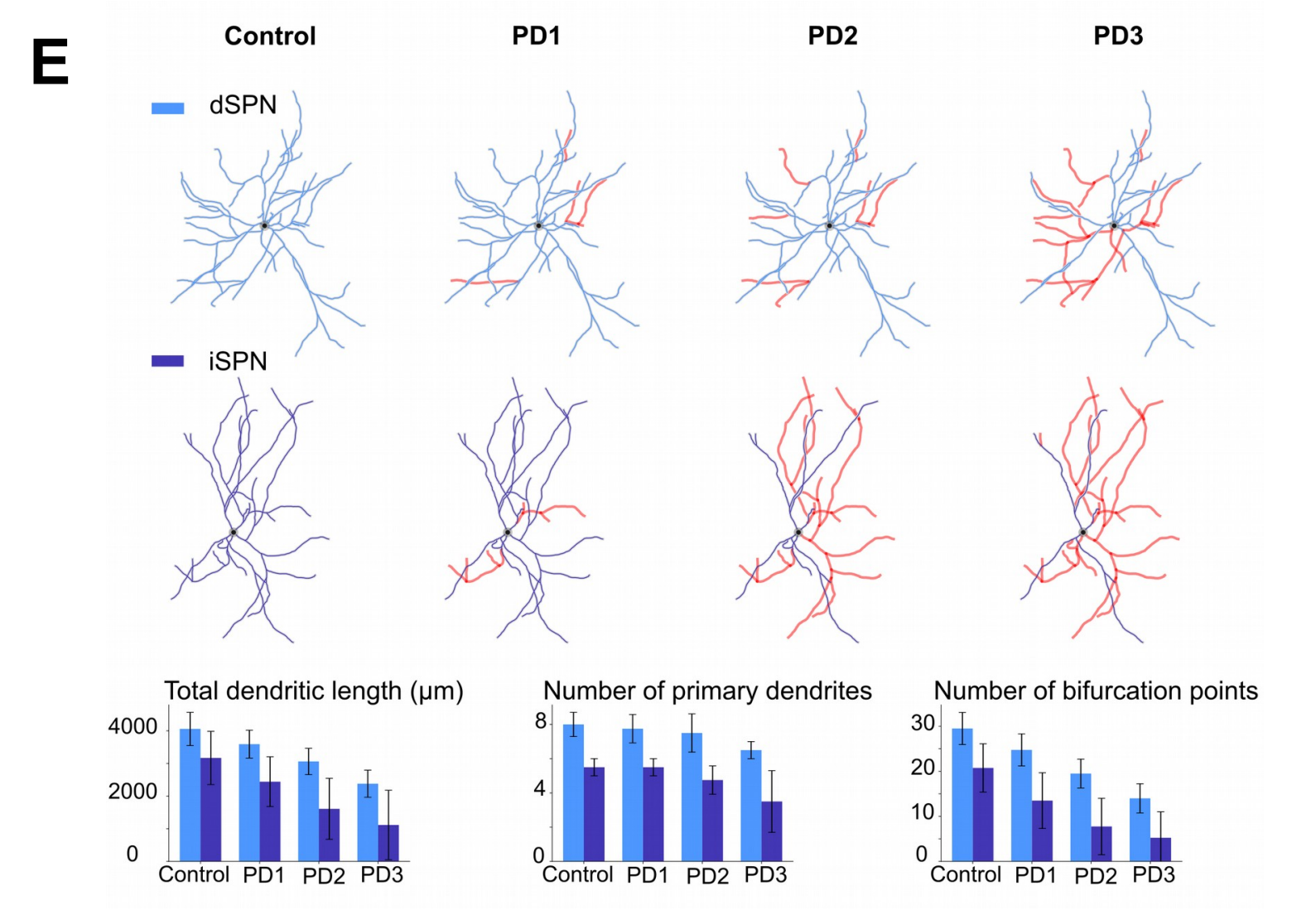

Dendritic atrophy and distribution of directed cliques We compared the distribution of topological motifs, in the form of directed cliques, between different microcircuits. This technique has already provided insight on structural and functional properties of the neocortical microcircuitry [4].
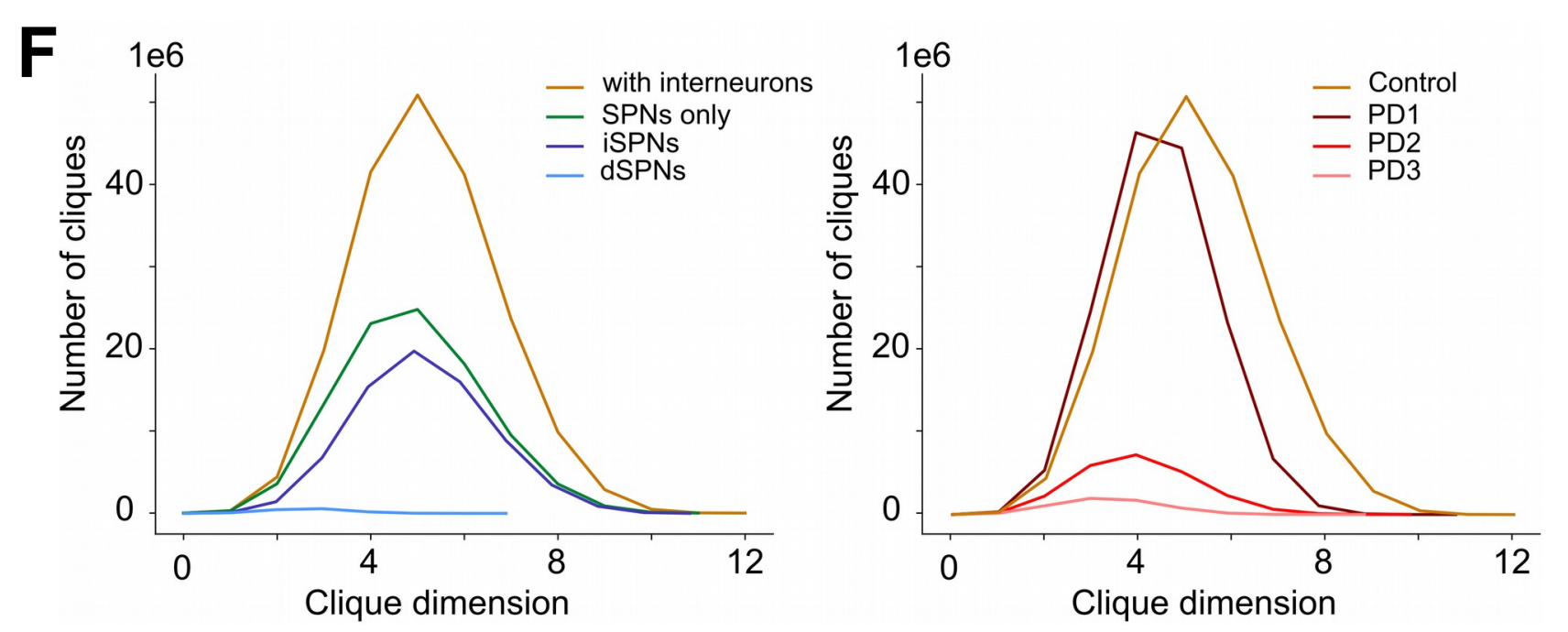

The distribution of directed cliques in the healthy striatal microcircuits (left image above) showed that striatal interneurons, despite only accounting for $5 \%$ of the total, are crucial for the construction of high dimensional directed cliques. Moreover there is a significant difference between networks made exclusively by dSPNs and iSPNs, as the former are extremely less likely to form cliques.

In PD networks (right image above) as expected, the presence of directed cliques drastically decreased with disease progression.

\section{Synaptic loss and striatal correlations}

A direct consequence of dendritic atrophy is loss of cortico-striatal inputs. To investigate how this affects the transfer of correlated input from cortex we simulated a pair of SPNs with realistic morphology from [5] using NEURON. Each SPN received excitatory input from cortex and inhibitory input from the local circuit. Correlated inputs were modelled as Multi-Interaction-Processes [6].

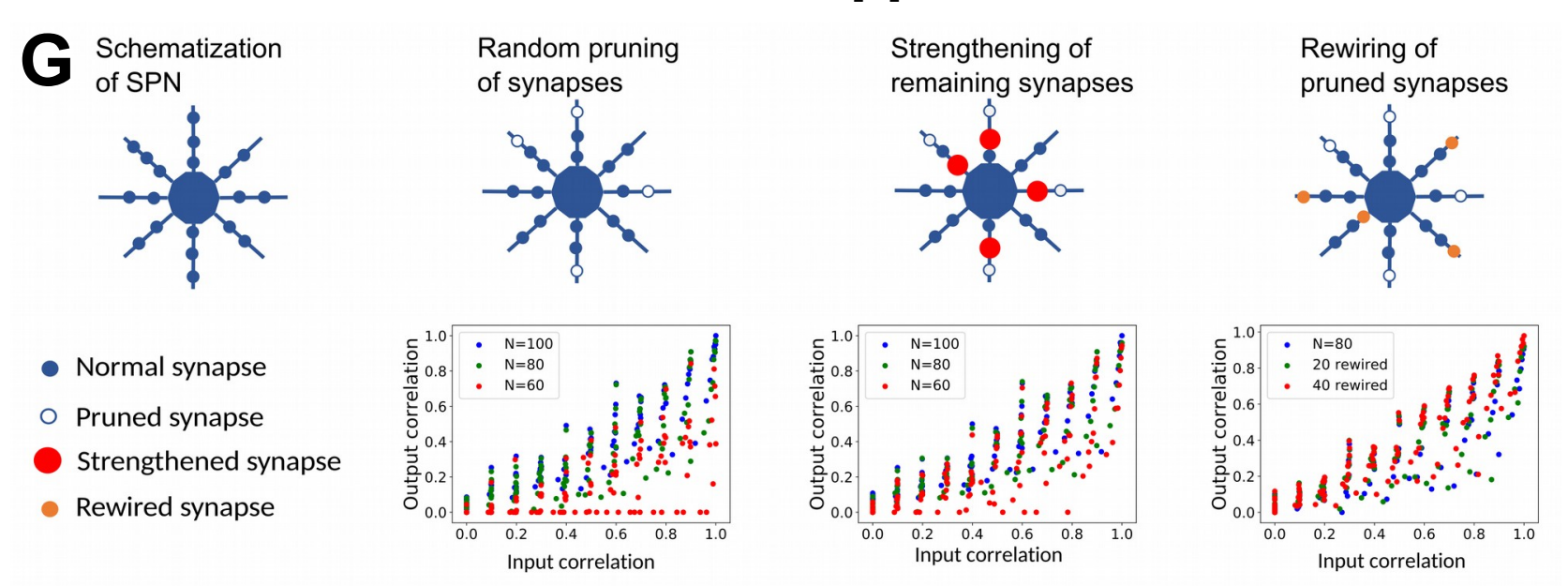

Normal levels of correlation transfer can not be recovered if existing synapses are strengthened to compensate for the loss of inputs. However, they can be recovered if cortical synapses are reallocated on the surviving dendrites.

- Striatal interneurons despite their low percentage are crucial for the construction of high dimensional cliques.

- dSPNs are less likely to form cliques than iSPNs.

- The presence of directed cliques drastically decreased with Parkinson's disease progression.

- Preliminary simulations suggested that the transmission of correlated input from cortex is decreased due to synaptic loss, which can be compensated by new distributed synapses.

- Future work will investigate how the presence of directed cliques affects the striatal network function in health and disease.

Acknowledgement: The simulations were enabled by resources provided by the Swedish National Infrastructure for Computing (SNIC) at the PDC Center for High Performance Computing, KTH Royal Institute of Technology, partially funded by the Swedish Research Council through grant agreement no. 2016-07213. The study was supported by the Swedish research council (VR-M-2017-02806), Swedish e-Science (SeRC). EU/Horizon 2020 no. 785907 (HBP SGA2) and no. 945539 (HBP SGA3).

\footnotetext{
- A directed graph $G$ is a pair $(V, E)$ where $V$ is a finite set of vertices (corresponding to neurons) and $E$ is a set of ordered pairs of distinct vertices $(\mathrm{v}, \mathrm{w})$ called edges (corresponding to synaptic connections).

- A vertex $v$ is a source (or a sink) if all edges including $v$ are from (or to) $v$.

A set $\left\{\mathrm{v}_{0}, \ldots, \mathrm{v}_{\mathrm{n}}\right\}$ of vertices is a $\mathrm{n}$-directed clique if it contains a source and a sink.
}
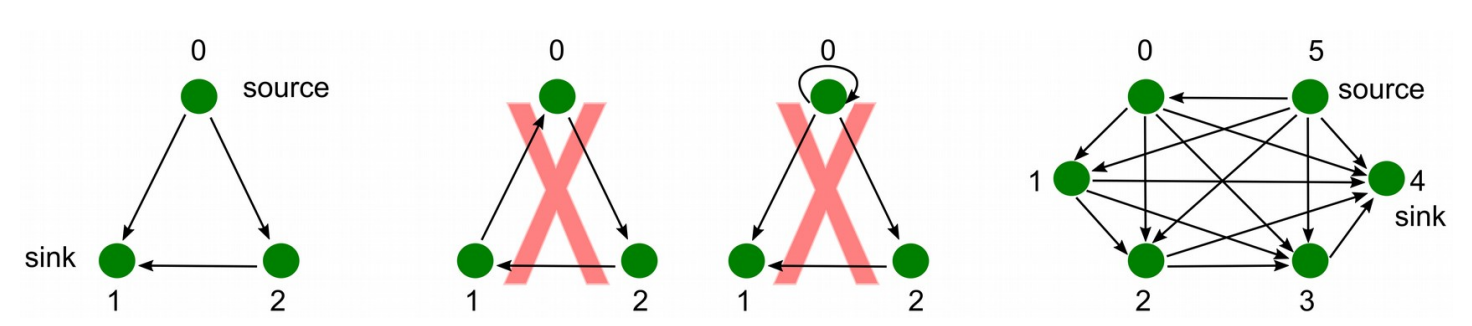